\title{
Identification and mapping of CpPM10.1, a major gene involved in powdery mildew (race 2 France of Podosphaera xanthi) resistance in zucchini (Cucurbita pepo L.)
}

\author{
Yunli Wang \\ Northeast Agricultural University \\ Cong Qi \\ Northeast Agricultural University \\ Yusong Luo \\ Northeast Agricultural University \\ Feng Zhang \\ Northeast Agricultural University \\ Zuyun Dai \\ Jianghuai horticulture Seeds Corporation limited \\ Man Li \\ Jianghuai Horticulture Seeds Corporation Limited \\ Shuping Qu ( $\nabla$ spqu@neau.edu.cn ) \\ Northeast Agricultural University ,Harbin, China https://orcid.org/0000-0003-4384-4425
}

\section{Research Article}

Keywords: Fine mapping, Powdery mildew resistant gene, Cucurbita pepo, race 2 France of Podosphaera xanthe RPW8 domain

Posted Date: February 12th, 2021

DOI: https://doi.org/10.21203/rs.3.rs-213194/v1

License: (c) (1) This work is licensed under a Creative Commons Attribution 4.0 International License. Read Full License 


\section{Abstract}

Powdery mildew (PM) is one of the most destructive fungal diseases, reducing the productivity of Cucurbita crops globally. In the present study, the zucchini inbred line 'X10' had highly stable PM resistance at the key time of inoculation ( $6 \mathrm{hpi}$ to $48 \mathrm{hpi}$ ), and 'Jin234' showed PM susceptible. Genetic analysis revealed that PM resistance in ' $\mathrm{X} 10$ ' is controlled by one major dominant locus, CpPM10.1 . Based on the strategy of QTL-seq combined with linkage analysis and the Kompetitive Allele-Specific PCR (KASP) method, CpPM10.1 was located in a $382.9 \mathrm{~kb}$ region on chromosome 10. Using 1,400 F 2 individuals and $\mathrm{F} 2: 3$ offspring of the recombinants, CpPM10.1 was defined as a region of approximately $20.9 \mathrm{~kb}$ that contained 5 coding genes. Among them, Cp4.1LG10g02780, Cp4.1LG10g02800 and Cp4.1LG10g02750 contained a RPW8 domain, which controls resistance to a broad range of PM pathogens. Nonsynonymous SNPs existed between 'X10' and 'Jin234' in Cp4.1LG10g02780 and Cp4.1LG10g02750 . Furthermore, the expression of Cp4.1LG10g02780 in 'X10' and the R-F2 pool was significantly higher than that in 'Jin234' and the S-F2 pool at the key period of inoculation, which showed that the expression of Cp4.1LG10g02780 was strongly positively involved in PM resistance. Further allelic diversity analysis in zucchini germplasm resources indicated that PM resistance associated with two SNPs in the RPW8 domain of Cp4.1LG10g02780. This study not only provides highly stable PM resistance gene resources in cucurbit crops but also lays the foundation for the functional analysis of PM resistance and resistance breeding in zucchini.

\section{Full Text}

Due to technical limitations, full-text HTML conversion of this manuscript could not be completed. However, the manuscript can be downloaded and accessed as a PDF.

\section{Figures}



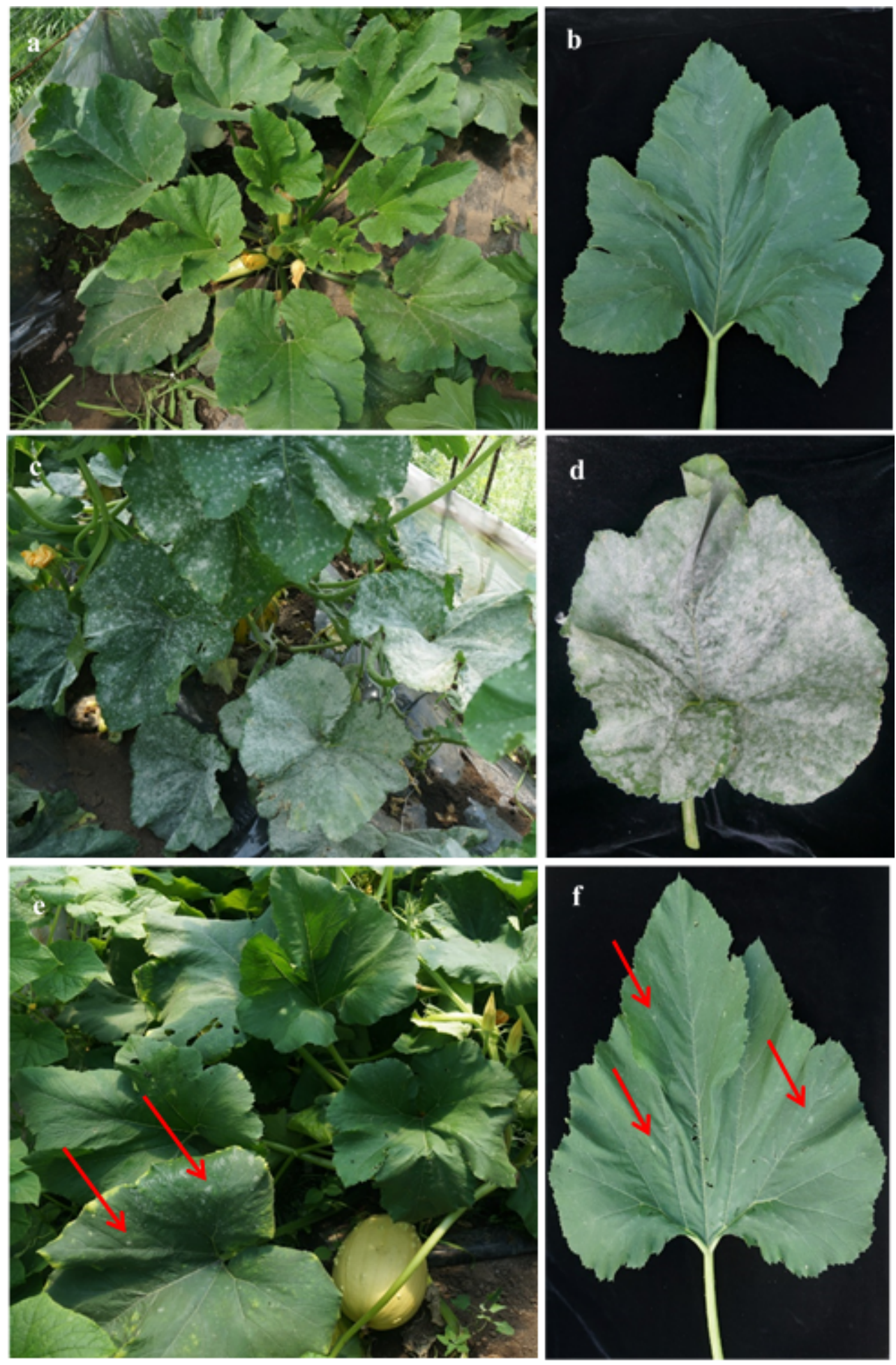

Figure 1

Identification of powdery mildew resistance of the adult parents and their Fi plants at $14 \mathrm{~d}$ after inoculation (a) Phenotype of 'X10' adult plant. (b) The fourth-leaf of 'X10' adult plant. (c) Phenotype of 'JIN23' adult plant. (d) The fourth-leaf of 'JIN23' adult plant. (e) Phenotype of F adult plant. (f) The fourth-leaf of F; adult plant. Red arrows indicated disease spot of powdery mildew. 


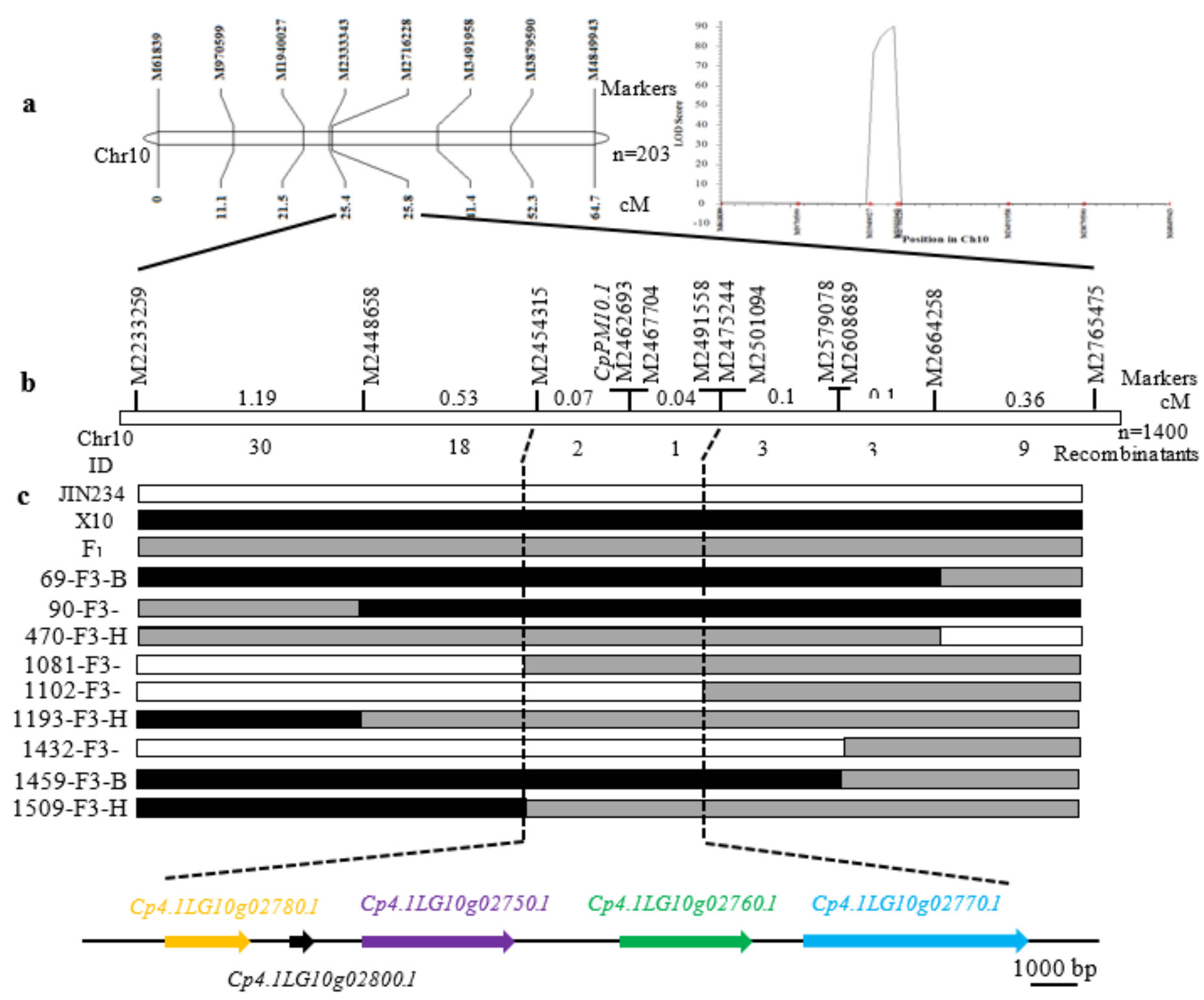

Figure 2

Genetic and physical mapping of CpPM10.1 (a) Genetic mapping of CpPM/0.] by KASP. Using the $203 \mathrm{~F}$; individuals from ' $X 10^{\circ} \mathrm{x}$ 'JIN234', CpPM10.1 locus was located to a $382.9 \mathrm{~kb}$ region between marker M2333343 and marker M2716228 on chromosome 10. (b) and (c). Fine mapping based on 1,400 F: plants and 66 recombinants with Phenotype of F2.; offsprings defined the CpPM1/0./ locus to a $20.9 \mathrm{~kb}$ region between marker M2454315 and marker M2475244. (d) Five coding genes predicted in the $20.9 \mathrm{~kb}$ region and the structure of these five genes. The boxes represent genes. 
a $\mathrm{X} 10$
JIN234

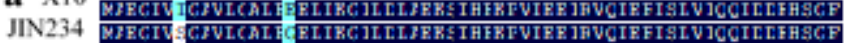

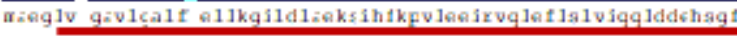

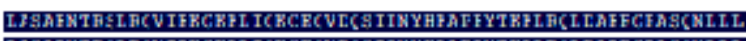

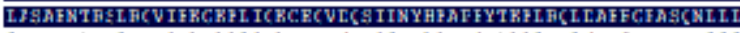

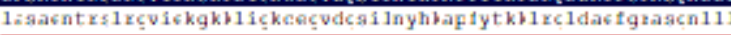

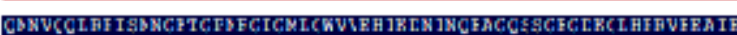

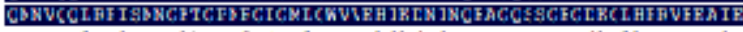

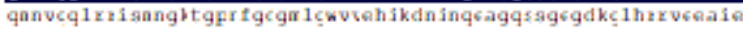

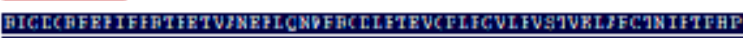

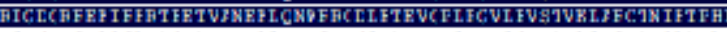

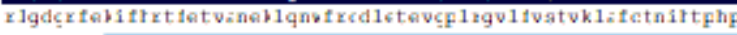

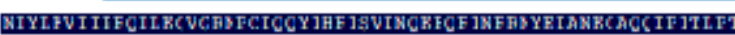
WIYLFVIIIFCIL RCVCFBFCICCY IHFISVIXCRECF INFHSYRIANKCACCIF]TLFT

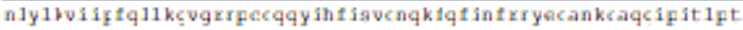
CIITCE CIHCF

b $\mathrm{x} 10$ tx:s:

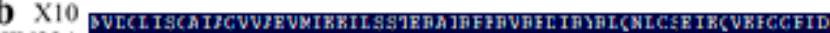

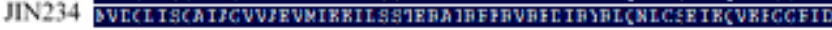

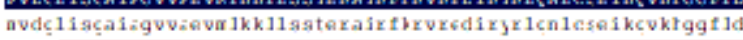

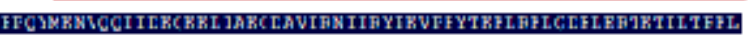

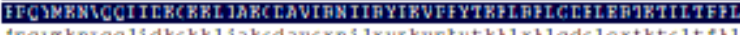

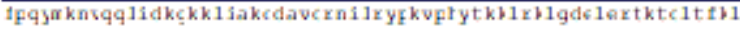

\section{EICSBT}

ICOSTT

a 1 qrat:

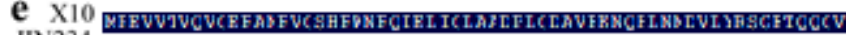

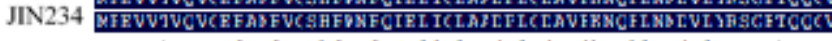

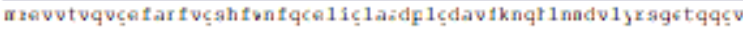

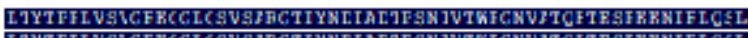

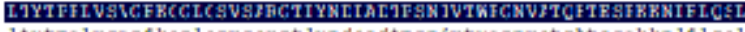

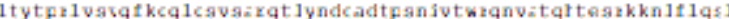

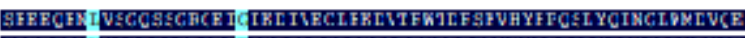

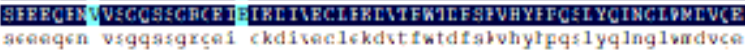

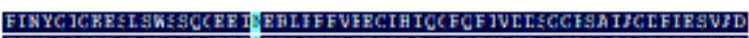

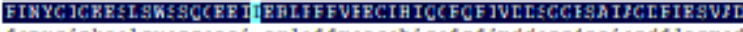

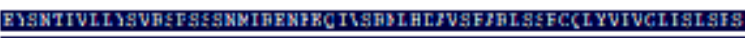

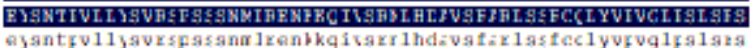

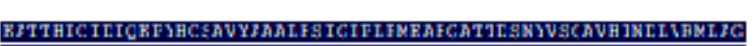

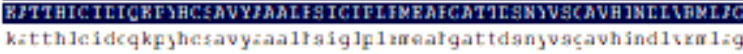

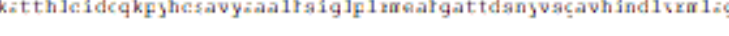

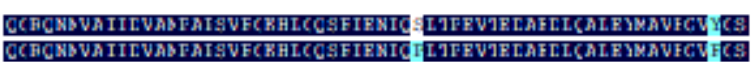

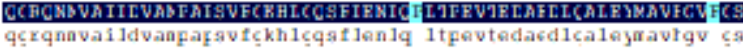

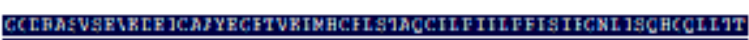

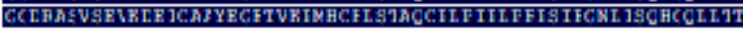

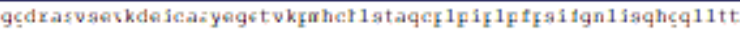

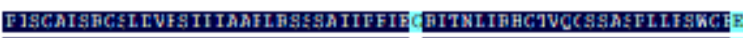

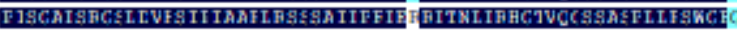

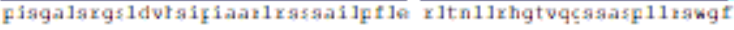

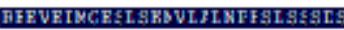

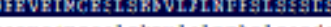

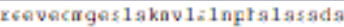

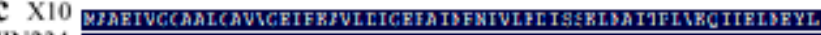

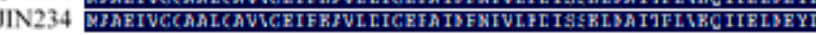

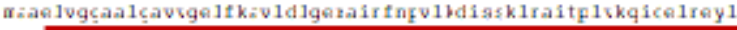

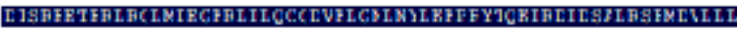

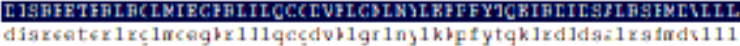

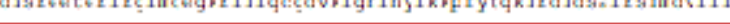

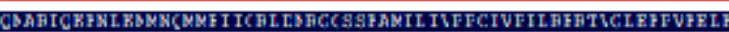

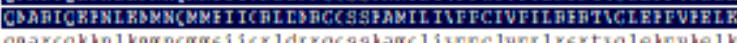

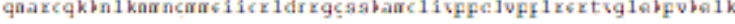

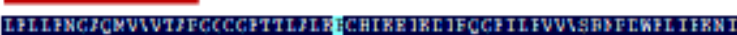

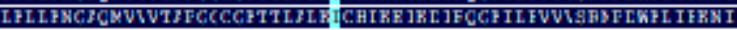

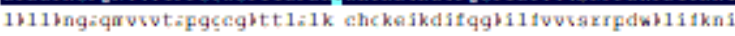

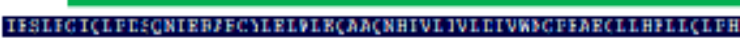

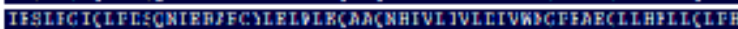

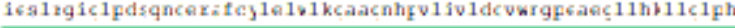

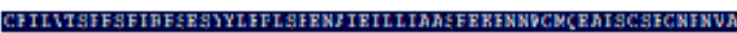

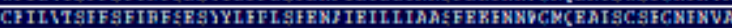

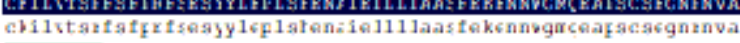

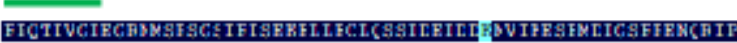

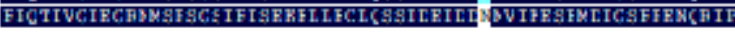

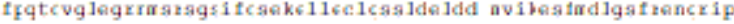

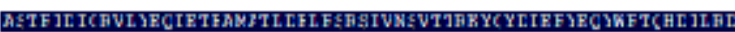

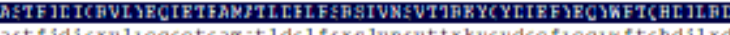

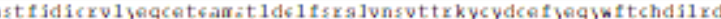

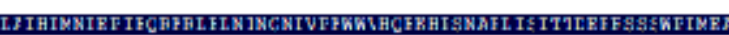

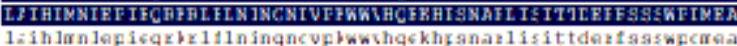

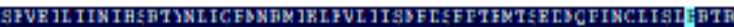

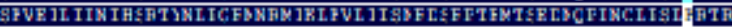

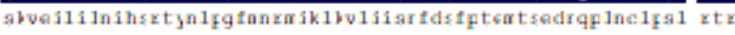

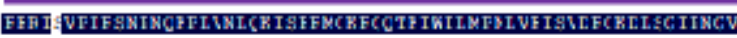

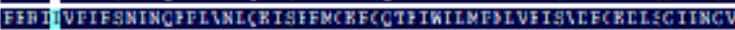

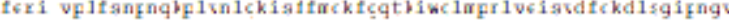

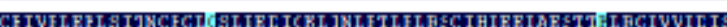

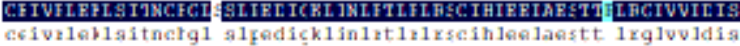

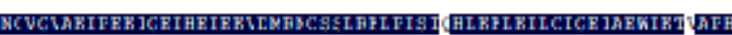

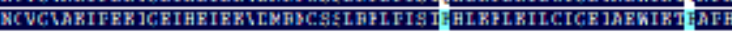

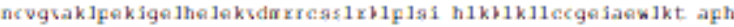

LFRCWVFCE TLFKLC

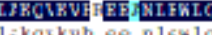

d X10

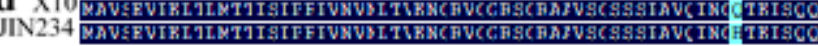

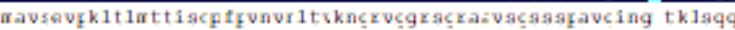

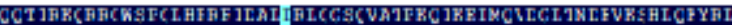

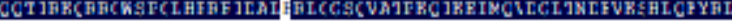

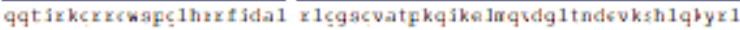

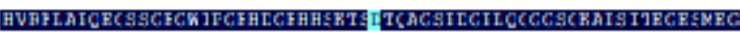

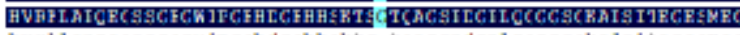

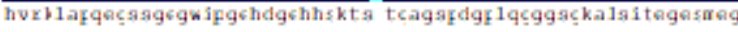

ERLFFII HEKRCF ICPHCD

ERLFRSI HESKRCF TCPHCD

คดdikad hrkkgriqhingd

\section{Figure 3}

Alignment of the predicted protein sequences of five genes between resistance ' $\mathrm{X} 10$ ' and susceptible 'JIN234' (a) Alignment acid sequences of Cp4.JLG10g02780. (b) Alignment acid sequences of Cp4.1LG10g02800. (c) Alignment acid sequences of Cp4.1ZLG/0g02750. (d) Alignment acid sequences of Cp4.1ZG10g02760. (e) Alignment acid sequences of Cp4.1LG10g02770. The red lines indicated RPW8 domains. The blue lines indicated GRAM domain. The green lines indicated NB-ARC domain the purple lines indicated leucine-rich repeat domains, the yellow lines indicated MYB domains 

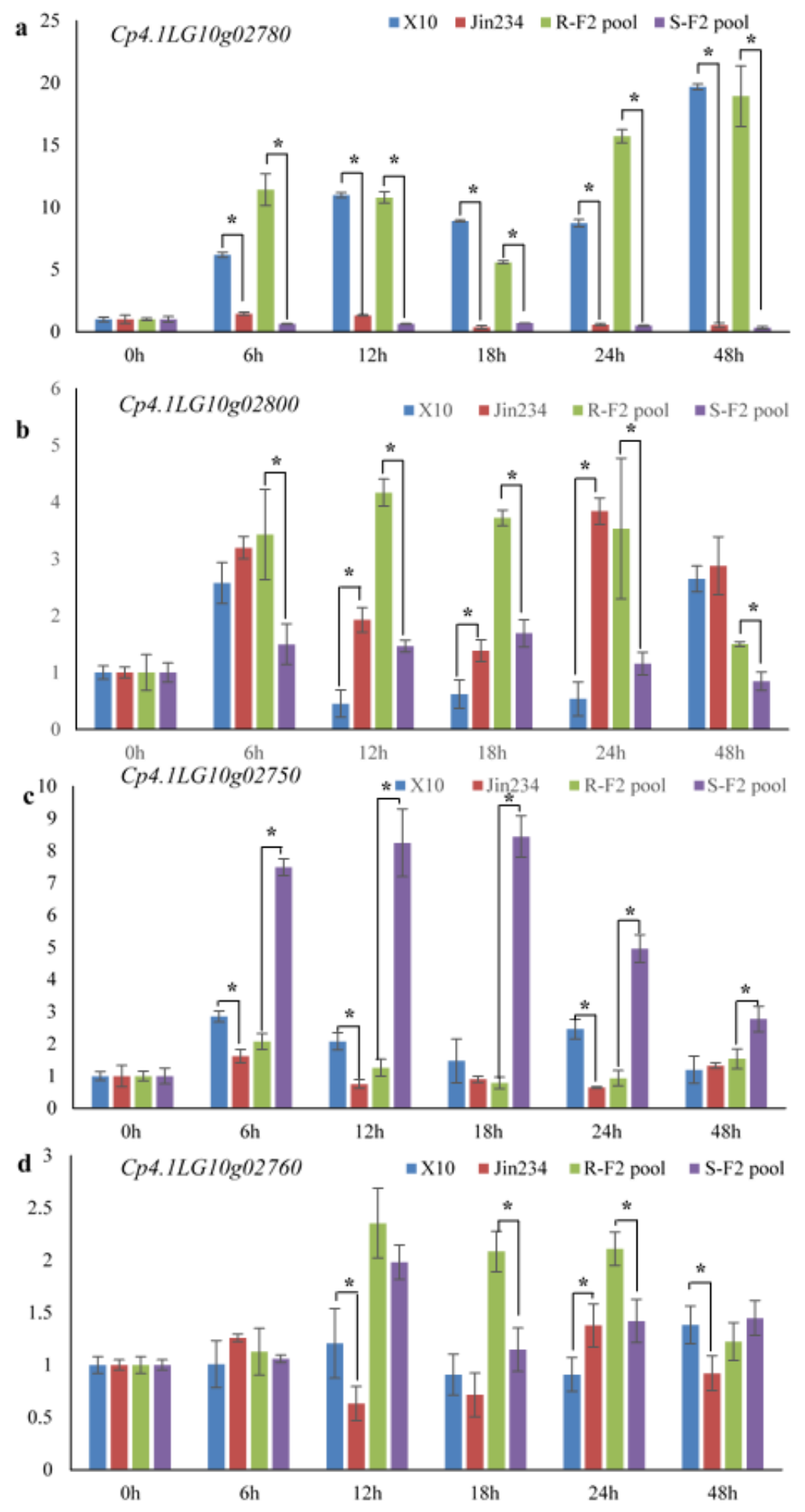

\section{Figure 4}

Relative expression levels of five candidate genes in resistance " $X 10$ *, susceptible 'JIN234', R-F2 pool and S-F2 pool (a) Expression levels of Cp4.1ZLG/0g02780. (b) Expression levels of Cp4./ZG10g02800. (c) Expression levels of Cp4.1LG10g02750. (d) Expression levels of Cp4.]ZG/0g02760. The relative expression levels of four genes were quantified using the $2^{\circ} 44 C T$ method (Livak and Schmittgen 2001). For the two parents and two pools, the expression level of the respective genes in $0 \mathrm{~h}$ after PM infection 
was set to a value of 1 and used as a reference. respectively. Each was repeated three times. and 15 plants were mixed in equal amounts to form one replicate in two pools. ${ }^{* \star}$ mdicates an extremely significant difference, $\mathrm{P}=0.01$; * indicates a significant difference, $\mathrm{P}=0.05$.

\begin{tabular}{|c|c|c|c|c|}
\hline ATG & \multicolumn{2}{|c|}{ Cp4.1LGIOg02780 } & TAA & \\
\hline 47 & & & & \\
\hline$(\mathrm{T}>\mathrm{G}) \quad(\mathrm{A}>\mathrm{C}$ & & & & \\
\hline$(\mathrm{I}-\mathrm{S}) \quad(\mathrm{E}-\mathrm{C}$ & & & & \\
\hline Accession ID & Taxon & Origin & Amino acid & DI \\
\hline $\mathrm{X} 10$ & C.pepo L. & Gansu,China & MAEGLVIGAVLGALFEEL & 0.5 \\
\hline $\mathrm{X} 16$ & C.pepoL. & Neimenggu, China & MAEGLVIGAVLGALFEEL & 0 \\
\hline Yun1m & C.pepo L. & Yunnan, China & MAEGLVIGAVLGALFEEL & 0 \\
\hline $711 \mathrm{~d}$ & C.pepo L. & Gansu,China & MAEGLVIGAVLGALFEEL & 0.5 \\
\hline $12689 \mathrm{~m}$ & C.pepo L. & Gansu,China & MAEGLVIGAVLGALFEEL & 0.5 \\
\hline $\mathrm{X} 7$ & C.pepo L. & Neimenggu, China & MAEGLVIGAVLGALFEEL & 1 \\
\hline $9 \mathrm{~d}$ & C.pepo L. & Henan, China & MAEGLVIGAVLGALFEEL & 0.5 \\
\hline Yun9d & C.pepoL. & Yunnan, China & MAEGLVIGAVLGALFEEL & 0.5 \\
\hline $\mathrm{A} 002-1$ & C.pepoL. & Yunnan, China & MAEGLVSGAVLGALFGEL & 1 \\
\hline $\mathrm{DL}$ & C.pepo L. & Gansu,China & MAEGLVSGAVLGALFGEL & 1 \\
\hline $\mathrm{X} 1$ & C.pepo L. & Neimenggu, China & MAEGLVSGAVLGALFGEL & 1 \\
\hline JIN234 & C.pepo L. & Heilongjiang, China & MAEGLVSGAVLGALFGEL & 5 \\
\hline $057 \mathrm{~m}$ & C.pepo L. & Gansu,China & MAEGLVSGAVLGALFGEL & 4.5 \\
\hline $3711 \mathrm{~m}$ & C.pepo L. & Gansu,China & MAEGLVSGAVLGALFGEL & 5 \\
\hline $1370 \mathrm{~m}$ & C.pepo L. & Gansu,China & MAEGLVSGAVLGALFGEL & 4 \\
\hline Yun6d & C.pepo L. & Yunnan, China & MAEGLVSGAVLGALFGEL & 4 \\
\hline GS36 & C.pepo L. & Gansu,China & MAEGLVSGAVLGALFGEL & 4.5 \\
\hline 0602 & C.pepo L. & Gansu, China & MAEGLVSGAVLGALFGEL & 4.5 \\
\hline Yun7d & C.pepo L. & Yunnan, China & MAEGLVSGAVLGALFGEL & 4 \\
\hline $\mathrm{GS} 24$ & C.pepo $\mathrm{L}$. & Gansu,China & MAEGLVSGAVLGALFGEL & 4 \\
\hline 235 & C.pepo $\mathrm{L}$. & Heilongjiang, China & MAEGLVSGAVLGALFGEL & 4 \\
\hline 234 & C.pepo L. & Heilongiang, China & MAEGLVSGAVLGALFGEL & 5 \\
\hline
\end{tabular}

\section{Figure 5}

Allelic variation in Cp4.1LG10g02 780 gene is associated with PM resistance in germplasm resources, Red letters indicates correspondence with the ammo acids in " $\mathrm{X} 10$ ', and green letters indicates 
correspondence with the amino acids in 'JIN234'.

\section{Supplementary Files}

This is a list of supplementary files associated with this preprint. Click to download.

- FigS1.pdf

- FigS2.pdf

- FigS3.pdf

- Figs4.pdf

- Figs5.emf

- Figs6.emf

- Tables1.pdf 